\title{
Tumor size assessment of invasive breast cancers: which pathological features affect MRI-pathology agreement?
}

\author{
Luciana Karla Lira França ${ }^{1}$, Almir Galvão Vieira Bitencourt ${ }^{1 *}$, Cynthia Aparecida Bueno de Toledo Osório², \\ Luciana Graziano ${ }^{1}$, Camila Souza Guatelli ${ }^{1}$, Juliana Alves Souza ${ }^{1}$ and Elvira Ferreira Marques ${ }^{1}$
}

\begin{abstract}
Background: Preoperative assessment of tumor size is important in breast cancer treatment planning, especially in breast conservation surgeries. The use of the Magnetic Resonance Imaging (MRI) is increasing among patients with newly diagnosed breast cancers. However, some pathological features can overestimate the measurement of tumor size by MRI, increasing mastectomy rates. The objective is to evaluate which pathological features may affect the agreement between MRI and pathologic tumor size on invasive breast carcinomas.

Methods: Eighty seven patients with breast cancer who underwent preoperative breast MRI were retrospectively evaluated. The main tumor size measured by MRI was compared with pathology (gold standard) and concordance was defined as a greater diameter difference of less than $10 \mathrm{~mm}$.

Results: There was MRI-pathology concordance in 60 cases (69.0\%), MRI overestimated tumor size in 21 (24.1\%) and underestimated in 6 (6.9\%). After multivariate analysis, only associated ductal carcinoma in situ (DCIS) remained significantly related to overestimation of tumor size on MRI (OR: 9.00; 95\% IC:1.13-71.87; $p=0.038$ ).

Conclusion: There was good correlation between tumor size evaluation on MRI and pathology. The presence of associated DCIS was the only pathological parameter associated with size overestimation on MRI.
\end{abstract}

Keywords: Breast cancer, Magnetic resonance imaging, Pathology surgical

\section{Background}

Preoperative assessment of tumor size is important in breast cancer treatment planning, especially in breast conserving surgeries. Preoperative Magnetic Resonance Imaging (MRI) has been used in patients with newly diagnosed breast cancer for proper evaluation of tumor extent. However, some pathological features can lead to an overestimation of tumor size by MRI, which consequently increases rates of mastectomy [1-3].

The exact reasons for tumor size overestimation by MRI are not entirely understood. Prior reports have demonstrated that the presence of high-risk breast lesions, benign epithelial proliferative lesions or specific morphologic findings such as satellite lesions, ductal

\footnotetext{
* Correspondence: almirgvb@yahoo.com.br

${ }^{1}$ Imaging Department, A.C.Camargo Cancer Center, R. Prof. Antonio

Prudente, 211, São Paulo, SP 09015-010, Brazil

Full list of author information is available at the end of the article
}

carcinoma in situ (DCIS), and/or lymphovascular invasion around the main tumor may cause MRI size overestimation [4]. Also, it is important to mention that tumor measurement on MRI may include both the invasive and non invasive components. In contrast, the pathologic examination of tumor size according to the TNM staging system is defined by the greatest diameter of the invasive breast carcinoma and DCIS extent is not taken into consideration [5].

Our hypothesis is that breast MRI is a good exam to predict pathologic tumor size, however, it can be affected by pathological parameters that will cause extent overestimation. The aim of this study was to evaluate which pathological features may influence the agreement on tumor size assessment of invasive breast cancers between MRI and pathology. 


\section{Methods}

After approval of the institution ethics review board, we retrospectively evaluated 87 patients with breast cancer who were submitted MRI for preoperative staging and treatment planning, from August 2012 to August 2014. Patients submitted to MRI or surgery at another institution were excluded. Mean time between MRI and surgery was 46 days. Data on tumor and patient characteristics were obtained from the original histopathologic, radiological and clinical reports. The size of the main tumor measured by MRI was compared to the tumor size described in the pathology report and defined as the greatest diameter of the invasive breast carcinoma (gold standard). Agreement between MRI and pathology results was defined as a greater diameter difference of less than $10 \mathrm{~mm}$, as used in prior studies [5]. Pathology of all discordant cases was reviewed.

Breast cancer histologic classification was based on the recommendations by the World Health Organization (WHO) Classification of Tumors of the Breast (4th Ed) and tumors were categorized as invasive breast cancer of no special type (NST), previously known as invasive ductal carcinoma (IDC), and special types of invasive breast carcinoma (i.e. invasive lobular carcinoma, tubular, mucinous, metaplastic carcinoma, etc) [6]. Immunohistochemical data was retrieved from pathology files and breast cancer molecular subtype was defined according to estrogen receptor (ER)/progesterone receptor (PR)/Her-2 status and Ki67 index, as follows: luminal A (expression of ER and/or PR, with Ki-67 <20\%); luminal B (expression of ER and/or PR, with Her-2 overexpression or Ki-67 $\geq 20 \%$ ); Her-2 overexpression (no expression of ER and PR, with Her-2 overexpression); and triple negative (no expression of ER, PR and Her-2) [7, 8].

MRI images were obtained with the patient in the prone position at a 1.5 Tesla equipment (Signa HDxT, GE), using dedicated 8-channel breast coil. Each exam consists of images taken before and after the use of paramagnetic contrast (gadolinium diethylenetriaminepentaacetic acid; Gd-DTPA). Before contrast administration, a T1 gradientecho sequence and a fat-saturated STIR sequence were acquired. Five gradient-echo axial 3D T1-weighted sequences were obtained using fat suppression for dynamic examination. The first sequence is obtained before contrast injection, the second after $20 \mathrm{~s}$ of contrast injection, and the others sequentially in the following minutes with a temporal resolution of 60-90 s. Post-processing subtraction images were obtained from pre and post-contrast sequences. The last sequence consists of gradient-echo sagittal 3D T1-weighted images. Main tumor size was measured on the largest diameter in the post-contrast sequence in which the lesion was more evident.

All data were stored in a database for statistical analysis using SPSS version 20.0. The descriptive analysis of categorical variables was calculated as simple and relative frequencies. Numerical variables were described as mean, standard deviation (SD), minimum and maximum. For univariate analysis, Pearson chi-square test with Yates correction or Fisher's exact test was used to compare categorical variables. For multivariate analysis, multiple logistic regression was performed, using the comparison of the size of the invasive tumor on MRI in relation to pathology (overestimated vs. not overestimated) as the dependent variable. The following pathological features were included as independent variables in the regression model: histological type, molecular subtype, presence of inflammatory infiltrate, desmoplastic reaction and associated DCIS. The odds ratio (OR) and $95 \%$ Confidence Interval (CI) were calculated for all variables and the level of significance adopted was $5 \%$.

\section{Results}

The mean age of patients was 53 years, range 30 to 81 years. The main tumor appeared on MRI as a mass in 72 cases $(82.8 \%)$, and non-mass enhancement in 15 (17.2\%). The most common histological type and molecular subtype were NST/IDC (57.5\%) and luminal B (51.2\%), respectively. Table 1 summarizes pathological findings of invasive carcinomas.

The largest dimension of the main tumor varied from 8 to $111 \mathrm{~mm}$ in MRI (mean: $32.4 \pm 21.9 \mathrm{~mm}$ ) and from 5 to $100 \mathrm{~mm}$ in pathology (mean: $26.6 \pm 19.5$ ). There was good agreement between the tumor size measured by

Table 1 Pathological findings of invasive carcinomas $(n=87)$

\begin{tabular}{ll}
\hline Pathological findings & $\mathrm{N}(\%)$ \\
\hline Histological Type & $50(57.5 \%)$ \\
NST invasive carcinomas & $14(16.1 \%)$ \\
Invasive lobular carcinomas & $23(26.4 \%)$ \\
Other special type carcinomas & \\
Associated DCIS & $63(72.4 \%)$ \\
Present & $24(27.6 \%)$ \\
Absent & \\
Desmoplastic Reaction & $75(86.2 \%)$ \\
Moderate / Intense & $12(13.8 \%)$ \\
Absent / Mild & \\
Inflammatory Infiltrate & $11(12.6 \%)$ \\
Moderate / Intense & $76(87.4 \%)$ \\
Absent / Mild & \\
Molecular Subtype & $32(36.8 \%)$ \\
Luminal A & $44(50.6 \%)$ \\
Luminal B & $4(4.6 \%)$ \\
Her-2 & $6(6.9 \%)$ \\
Triple-negative &
\end{tabular}

NST no special type 
MRI and pathology in 60 cases (69.0\%), MRI overestimated tumor size in 21 (24.1\%) and underestimated in 6 (6.9\%).

Table 2 describes the correlation between pathological features and overestimation of tumor size on MRI at univariate analysis. After multivariate analysis, only associated DCIS remained significantly related to overestimation of tumor size on MRI (OR: 9.00; 95\% IC: 1.13-71.87; $p=0.038$ ).

\section{Discussion}

MRI is often used, in addition to conventional imaging, for the preoperative assessment of breast cancer size to determine the optimal surgical strategy. Recent studies have suggested that MRI is superior to mammography and ultrasound in determining invasive tumor size, depicting multifocality, as well as evaluating the intraductal component $[1-5,9]$.

Despite substantial improvement in breast MRI technique and interpretation, true tumor size can be over or underestimated in some patients, which may change treatment decisions. In our sample population MRI overestimated the pathology size in $24 \%$ of the cases, which is consistent with the literature, ranging from $6 \%$ to $81 \%$ [2, 10]. However, it should be remembered that for TNM classification, pathologists consider just the invasive component size of

Table 2 Correlation between pathological findings and overestimation of tumor size on MRI $(n=87)$

\begin{tabular}{llll}
\hline Pathological findings & \multicolumn{2}{c}{ MRl tumor size } & $p$ \\
\cline { 2 - 3 } & Overestimated & Not overestimated & \\
\hline Histological Type & & 0.172 \\
NST invasive carcinomas & $9(17.6 \%)$ & $42(82.4 \%)$ & \\
Invasive lobular carcinomas & $3(21.4 \%)$ & $11(78.6 \%)$ & \\
Other special type carcinomas & $8(38.1 \%)$ & $13(61.9 \%)$ & \\
Associated DCIS & & & 0.012 \\
Present & $19(30.2 \%)$ & $44(69.8 \%)$ & \\
Absent & $1(4.3 \%)$ & $22(95.7 \%)$ & \\
Desmoplasic Reaction & & & 0.670 \\
Moderate / High & $18(24 \%)$ & $57(76 \%)$ & \\
Absent / Mild & $2(18.2 \%)$ & $9(81.8 \%)$ & \\
Inflammatory Infiltrate & & & \\
Moderate / High & $19(25.3 \%)$ & $56(74.7 \%)$ & \\
Absent / Mild & $1(9.1 \%)$ & $10(90.9 \%)$ & \\
Molecular Subtype & & & \\
Luminal A & $6(19.4 \%)$ & $25(80.6 \%)$ & \\
Luminal B & $11(25 \%)$ & $33(75 \%)$ & \\
Her-2 & $2(50 \%)$ & $2(50 \%)$ & \\
Triple-negative & $0(0 \%)$ & $5(100 \%)$ & \\
\hline
\end{tabular}

MRI magnetic resonance imaging, NST no special type the tumor, while it is well established that MRI measurement includes both invasive and noninvasive components $[5,11,12]$.

Presence of associated carcinoma in situ was the only pathological finding associated with tumor size overestimation at MRI in our sample. MRI is useful in detecting DCIS, especially high grade, even in cases with negative mammography [13]. Mennella et al. studied the effect of tumor histology on the MRIpathology discordance and found that patients with DCIS had a higher median overestimation on MRI than all the other histological groups [14]. These authors also showed that the median overestimation in the patients who had IDC with extensive intraductal component was significantly higher than that of the patients who had IDC without extensive intraductal component [14]. Rominger et al. found that nonmass enhancement, which is commonly associated with DCIS, significantly predicted MRI-pathology discordance on tumor size [5]. Jethava et al. found that several factors affect the accuracy of MRI in the preoperative assessment of tumor size [15]. In that study, the most important factors associated to overestimation of tumor size on MRI were high-grade tumor (when compared to low and intermediate tumors) and DCIS (when compared to invasive carcinomas) [15].

The results of this study should be considered in the context of its limitations. Because of the retrospective nature of this study, it was not possible to measure the non invasive component of the tumors at pathology and compare it with the imaging size. Future prospective studies could compare macroscopic and microscopic pathologic evaluation with tumor size on MRI.

\section{Conclusions}

In conclusion, there was good correlation between the measurement of tumor on MRI and pathology. In 24\% of the patients, MRI overestimated tumor size and the presence of DCIS was the only pathological feature significantly associated with MRI-pathology discordance. These findings support that different methods of measurement are the main cause of MRI-pathology discordance on tumor size. While the pathologic TNM staging system defines tumor size according to the extent of the invasive carcinoma without taking DCIS in consideration, MRI demonstrates the extension of disease by estimating both the in situ and invasive components of the disease. Thus, these findings should be considered before stating that there is disagreement between the tumor size on MRI and surgical specimen, both in clinical practice and in scientific papers, to avoid misdiagnosis and hasty conclusions, which may impact patient management. 


\section{Abbreviations}

Cl: Confidence interval; DCIS: Ductal carcinoma in situ; ER: Estrogen receptor; IDC: Invasive ductal carcinoma; MRI: Magnetic resonance imaging; NST: No special type; OR: Odds ratio; PR: Progesterone receptor

\section{Acknowledgements}

Not applicable.

\section{Funding}

No funding received.

\section{Availability of data and materials}

The datasets analysed during the current study available from the corresponding author on reasonable request.

\section{Authors' contributions}

LKLF and AGVB designed the research. LKLF, AGVB, CABTO, LG, CSG, JAS and EFM performed the research and analyzed the data. All authors wrote/ revised the paper. All authors read and approved the final manuscript.

\section{Ethics approval and consent to participate}

This project received approval from the institution's Research Ethics Committee (no. 2062/15).

\section{Consent for publication}

Not applicable

\section{Competing interests}

None. All procedures performed in these study were in accordance with the ethical standards of the institutional research committee and with the 1964 Helsinki declaration and its later amendments.

\section{Publisher's Note}

Springer Nature remains neutral with regard to jurisdictional claims in published maps and institutional affiliations.

\section{Author details}

${ }^{1}$ Imaging Department, A.C.Camargo Cancer Center, R. Prof. Antonio Prudente, 211, São Paulo, SP 09015-010, Brazil. 'Pathology Department, A.C.Camargo Cancer Center, R. Prof. Antonio Prudente, 211, São Paulo, SP 09015-010, Brazil.

Received: 10 November 2017 Accepted: 12 January 2018

Published online: 01 February 2018

\section{References}

1. Hata T, Takahashi H, Watanabe K, Takahashi M, Taguchi K, Itoh T, et al. Magnetic resonance imaging for preoperative evaluation of breast cancer: a comparative study with mammography and ultrasonography. J Am Coll Surg. 2004:198:190-7.

2. Chandwani S, George PA, Azu M, Bandera EV, Ambrosone CB, Rhoads $G G$, et al. Role of preoperative magnetic resonance imaging in the surgical management of early-stage breast cancer. Ann Surg Oncol. 2014;21:3473-80.

3. Lai HW, Chen DR, Wu YC, Chen CJ, Lee CW, Kuo SJ, et al. Comparison of the diagnostic accuracy of magnetic resonance imaging with Sonography in the prediction of breast cancer tumor size: a concordance analysis with Histopathologically determined tumor size. Ann Surg Oncol. 2015;22(12): 3816-23.

4. Grimsby GM, Gray R, Dueck A, Carpenter S, Stucky CC, Aspey H, et al. Is there concordance of invasive breast cancer pathologic tumor size with magnetic resonance imaging? Am J Surg. 2009;198(4):500-4.

5. Rominger M, Berg D, Frauenfelder T, Ramaswamy A, Timmesfeld N. Which factors influence MRI-pathology concordance of tumour size measurements in breast cancer? Eur Radiol. 2016;26:1457-65.

6. Lakhani S, Ellis I, Schnitt S, Tan PH, van de Vijver MJ. WHO classification of Tumours of the breast. 4th ed. Lyon: IARC Press; 2012.

7. Coates AS, Winer EP, Goldhirsch A, Gelber RD, Gnant M, Piccart-Gebhart M, et al. Tailoring therapies-improving the management of early breast cancer: St Gallen international expert consensus on the primary therapy of early breast cancer 2015. Ann Oncol. 2015;26:1533-46.
8. Curigliano G, Burstein HJ, P Winer E, Gnant M, Dubsky P, Loibl S, et al. Deescalating and escalating treatments for early-stage breast cancer: the St. Gallen international expert consensus conference on the primary therapy of early breast cancer 2017. Ann Oncol. 2017:28:1700-12.

9. Liberman L, Morris EA, Dershaw DD, Abramson AF, Tan LK. MR imaging of the ipsilateral breast in women with percutaneously proven breast cancer. AJR Am J Roentgenol. 2003;180(4):901-10.

10. Behjatnia B, Sim J, Bassett LW, Moatamed NA, Apple SK. Does size matter? Comparison study between MRI, gross, and microscopic tumor sizes in breast cancer in lumpectomy specimens. Int J Clin Exp Pathol. 2010;3(3): 303-9.

11. Jansen SA, Newstead GM, Abe H, Shimauchi A, Schmidt RA, Karczmar GS. Pure Ductal carcinoma in situ: kinetic and morphologic MR characteristics compared with mammographic appearance and nuclear grade. Radiology. 2007:245:684-91.

12. Mossa-Basha M, Fundaro GM, Shah BA, Ali S, MV P. Ductal carcinoma in situ of the breast: MR imaging findings with histopathologic correlation. Radiographics. 2010;30:1673-87.

13. Kuhl CK, Schrading S, Bieling HB, Wardelmann E, Leutner CC, Koenig R, et al. MRI for diagnosis of pure ductal carcinoma in situ: a prospective observational study. Lancet. 2007;370:485-92.

14. Mennella S, Garlaschi A, Paparo F, Perillo M, Celenza M, Massa T, et al. Magnetic resonance imaging of breast cancer: factor affecting the accuracy of preoperative lesion sizing. Acta Radiol. 2015;56:260-88,

15. Jethava A, Ali S, Wakefield D, Crowell R, Sporn J, Vrendenburgh J. Diagnostic accuracy of MRI in predicting breast tumor size: comparative analysis of MRI vs Histopathological assessed breast tumor size. Conn Med. 2015;79:261-7.

\section{Submit your next manuscript to BioMed Central} and we will help you at every step:

- We accept pre-submission inquiries

- Our selector tool helps you to find the most relevant journal

- We provide round the clock customer support

- Convenient online submission

- Thorough peer review

- Inclusion in PubMed and all major indexing services

- Maximum visibility for your research

Submit your manuscript at www.biomedcentral.com/submit 